\title{
Curcumin Promotes Collagen Type I, Keratinocyte Growth Factor-1, and Epidermal Growth Factor Receptor Expressions in the In Vitro Wound Healing Model of Human Gingival Fibroblasts
}

\author{
Auspreeya Rujirachotiwat ${ }^{1,2}$ Supaporn Suttamanatwong $3,4, \odot$
}

${ }^{1}$ Department of Pediatric Dentistry, Faculty of Dentistry, Chulalongkorn University, Bangkok, Thailand

2Division of Dentistry, Banphue Hospital, Banphue District, Udonthani, Thailand

${ }^{3}$ Department of Physiology, Faculty of Dentistry, Chulalongkorn University, Bangkok, Thailand

${ }^{4}$ Research Unit of Herbal Medicine and Natural Product for Dental Treatment, Faculty of Dentistry, Chulalongkorn University,

Bangkok, Thailand

Eur J Dent:2021;5:63-70
Address for correspondence Supaporn Suttamanatwong, DDS, PhD, Department of Physiology, Faculty of Dentistry, Chulalongkorn University, Bangkok 10330, Thailand (e-mail: supaporn0067@gmail.com).

\begin{abstract}
Objective Curcumin promotes oral wound healing; however, the underlying mechanism remains unknown. We hypothesized that curcumin may regulate gene expression in human gingival fibroblasts (hGFs). This study investigated the effect of curcumin on the expression of wound healing-related genes, collagen type I (COL1), keratinocyte growth factor (KGF)-1, and epidermal growth factor receptor (EGFR), in the in vitro wound healing model of hGFs, as well as the signaling pathway involved in the regulation of these genes by curcumin.

Materials and Methods The hGFs were treated with curcumin in the unwounded condition and in the in vitro wound healing model (scratch assay). Gene expression was determined by quantitative polymerase chain reaction. PD98059 was used to elucidate whether extracellular signal regulated kinase (ERK) signaling is involved in the curcumin-regulated gene expression in hGFs. Cell migration was also analyzed by the scratch assay.

Statistical Analysis Data were analyzed by independent $t$-test or one-way analysis of variance (ANOVA) followed by Tukey's Honestly Significant Difference (HSD) test.

Results In unwounded hGFs, curcumin significantly increased KGF-1 and EGFR expressions but not COL1 mRNA expression. Interestingly, curcumin significantly upregulated COL1, KGF-1, and EGFR expressions in the in vitro wound healing model. Furthermore, PD98059 significantly decreased the curcumin-induced COL1 and EGFR

Keywords

- curcumin

- gingival fibroblasts

- collagen

- KGF-1

- EGFR expressions, but did not significantly affect KGF-1 upregulation by curcumin. However, hGF migration was not affected by curcumin treatment.

Conclusion Curcumin induced KGF-1 and EGFR expressions in unwounded hGFs. In the in vitro wound healing model, curcumin upregulated COL1 and EGFR expression via the ERK pathway and increased KGF-1 expression, possibly by an ERK-independent mechanism.
\end{abstract}

DOI https://doi.org/ $10.1055 / \mathrm{s}-0040-1715781$ ISSN $1305-7456$.

\section{(c) 2020. European Journal of Dentistry.}

This is an open access article published by Thieme under the terms of the Creative Commons Attribution-NonDerivative-NonCommercial-License, permitting copying and reproduction so long as the original work is given appropriate credit. Contents may not be used for commercial purposes, or adapted, remixed, transformed or built upon. (https://creativecommons.org/licenses/by-nc-nd/4.0/)

Thieme Medical and Scientific Publishers Pvt. Ltd., A-12, 2nd Floor, Sector 2, Noida-201301 UP, India 


\section{Introduction}

Oral wound healing is a complicated biological process that requires signals from different cell types, including keratinocytes, endothelial cells, immune cells, and fibroblasts. ${ }^{1}$ Generally, the wound healing process consists of four overlapping stages, that is, hemostasis, inflammation, proliferation, and remodeling. ${ }^{1}$ Gingival fibroblasts (GFs) play a key role in the proliferative phase by releasing several growth factors such as keratinocyte growth factor (KGF)-1 and transforming growth factor- $\beta$, which exhibit autocrine and paracrine effects to promote wound healing. Furthermore, GFs are responsible for synthesis of extracellular matrix (ECM) during granulation tissue formation for subsequent wound remodeling. Type-I collagen (COL1) is a major protein in the extracellular matrix that serves as a structural scaffold in many tissues. ${ }^{2}$ When tissues are disrupted following injury, fibroblasts are responsible for collagen synthesis, thus providing an extracellular matrix framework for cell adhesion and migration during wound healing. ${ }^{3}$

Keratinocyte growth factor-1 (KGF-1) or fibroblast growth factor-7 (FGF-7) is an important growth factor produced mainly by fibroblasts and acts specifically in a paracrine fashion on keratinocytes. ${ }^{4}$ KGF-1 expression is upregulated shortly after a wound occurs, suggesting that this growth factor is involved in the early phases of the repair process. ${ }^{4} \mathrm{KGFs}$ promotes reepithelialization by increasing keratinocyte proliferation and migration. ${ }^{4}$

Epidermal growth factor (EGF), first discovered in the salivary gland of a mouse, is produced mainly by parotid salivary glands in human with a higher concentration in saliva than in plasma. ${ }^{1}$ Increased level of EGF in saliva during oral wound healing suggests the important role of EGF in repair of oral mucosa. ${ }^{1,5}$ At wound site, platelets, macrophages, and fibroblasts are the source of EGF which stimulates wound closure. ${ }^{6}$ EGF binds to epidermal growth factor receptor (EGFR) expressed on many cell types including fibroblasts and epithelial cells. ${ }^{7}$ EGFR signaling is essential in wound healing by regulating migration, proliferation, and differentiation of epithelial and mesenchymal cells, as well as collagen deposition and granulation tissue formation. ${ }^{1,7,8}$

Curcumin (diferuloylmethane), a yellow substance in turmeric rhizome, belongs to a family of chemicals known as curcuminoids. ${ }^{9}$ Curcumin has been used as a spice and a powerful medicinal herb because of its anti-inflammatory, antioxidant, antibacterial, and anticarcinogenic effects. ${ }^{10-12}$ In addition, curcumin has been reported to promote healing in different types of dermal and oral wounds. ${ }^{13-15}$ Curcumin enhances dermal wound healing in part by stimulating collagen production and reducing oxidative stress. ${ }^{13}$ However, little is known about the mechanism by which curcumin promotes oral wound healing. Curcumin regulates expression of several proteins, growth factors, and inflammatory cytokines in many cell types..$^{16}$ The effect of curcumin in regulating gene expression in hGFs, however, remains unclear. We hypothesized that curcumin may regulate the expression of genes involved in wound healing in hGFs. Therefore, the aim of this study was to investigate the effect of curcumin on the expression of wound healing-related genes, COL1, KGF-1, and EGFR, in unwounded hGFs and in the in vitro wound healing model. Furthermore, the signaling pathway by which curcumin regulates these genes was also elucidated.

\section{Materials and Methods}

\section{Cell Culture}

HGFs were isolated from healthy gingival tissue explants from three donors (two males and 1 female; mean age, 21.3 years) who were undergoing surgical removal of third molars at the Department of Surgery, Faculty of Dentistry, Chulalongkorn University. Signed consent forms were obtained from the patients. The study protocol was approved by the Ethics Committee of the Faculty of Dentistry, Chulalongkorn University.

The gingival tissues were immediately transferred in the ice-cold Dulbecco's modified Eagle's medium (DMEM; Sigma, United States) consisting of $10 \%$ fetal bovine serum (FBS, Gibco, United States), 1\% L-glutamine (Gibco, United States), and 1\% antibiotic-antimycotic (Gibco, United States). The specimens were washed twice with phosphate-buffered saline (Gibco, United States). The collected gingival tissues were cut into $1 \mathrm{~mm} \times 1 \mathrm{~mm}$ pieces and placed in 60-mm dishes, and cultured in DMEM containing 10\% FBS, $1 \%$ L-glutamine, and $1 \%$ antibiotic-antimycotic at $37^{\circ} \mathrm{C}$ in a humidified $5 \%$ carbon dioxide atmosphere. After reaching confluence, the cells were subcultured using $0.125 \%$ trypsin (Gibco, United States) at a 1:3 ratio. The medium was renewed every 2 days. Primary human GFs (hGFs) from the third to the fifth passage were used in the experiments.

To investigate the effect of curcumin on gene expression, hGFs were seeded at $6 \times 10^{5}$ cells per $60-\mathrm{mm}$ dish in DMEM with $10 \% \mathrm{FBS}, 1 \% \mathrm{~L}$-glutamine, and $1 \%$ antibiotic-antimycotic. The next day, the cells were washed and switched to serumfree DMEM for 24 hours. The cells were then treated with $0,0.1,1,10$, or $20 \mu \mathrm{M}$ curcumin (Sigma, United States) for 24 hours. The cells were harvested for total RNA extraction and gene expression was determined by quantitative polymerase chain reaction (PCR).

\section{In vitro Wound Healing Assay (Scratch Assay)}

To determine the effect of curcumin on gene expression in wounded GFs, a scratch assay was performed to mimic a scratch wound. ${ }^{17}$ Briefly, hGFs were seeded at $6 \times 10^{5}$ cells per $60-\mathrm{mm}$ dish in DMEM with $10 \%$ FBS. On the following day, the cells were washed and switched to serum-free DMEM for 24 hours. When the cells reach confluence, a sterilized pipette tip $(200 \mu \mathrm{L})$ was used to create a straight scratch line across the cell monolayer. After the wounding, the media was removed and the cells were treated with dimethyl sulfoxide (DMSO) or $1 \mu \mathrm{M}$ curcumin for 24 hours. Then, the cells were harvested for total RNA extraction and gene expression was determined by quantitative PCR. To determine the role of ERK signaling in the curcuminregulated gene expression, the cells were incubated 
with dimethyl sulfoxide (DMSO) or a MEK/ERK signaling inhibitor, PD98059, (100 nM) (Cell signaling technology, United States) for 1 hour prior to vehicle (DMSO) or $1 \mu \mathrm{M}$ curcumin treatment for 24 hours.

\section{RNA Extraction and Quantitative Polymerase Chain Reaction}

Total RNA was extracted using a Total RNA Mini Kit (Geneaid, Taiwan) according to the manufacturer's instruction. The RNA concentration was determined by measuring the absorbance at 260 and $280 \mathrm{~nm}$ with a Thermo Scientific NanoDrop 2000 Spectrophotometer. A total RNA of $2 \mu \mathrm{g}$ of each sample was reverse transcribed to single-strand cDNA using Moloney Murine Leukemia Virus Reverse Transcriptase (M-MLV RT; Promega, United States) following the manufacturer's instruction.

The cDNA template was amplified using iTaq Universal SYBR Green Supermix (Bio-Rad, United States). The mixture contained $5 \mu \mathrm{L}$ iTaq Universal SYBR Green Supermix (2X), $0.25 \mu \mathrm{L}$ primer, and $2.5 \mu \mathrm{L}$ DNA template. Nucleasefree water was added to a final volume of $10 \mu \mathrm{L}$. The PCR program was $95^{\circ} \mathrm{C}$ for 5 minutes followed by 45 cycles for the amplification phase; each cycle consisted of denaturation for 30 seconds at $95^{\circ} \mathrm{C}$, annealing for 30 seconds at $56^{\circ} \mathrm{C}$ for glyceraldehyde-3-phosphate dehydrogenase (GAPDH), and $50^{\circ} \mathrm{C}$ for other genes, and extension for 30 seconds at $72^{\circ} \mathrm{C}$. The primer sequences used for PCR amplification are shown in - Table 1. All quantitative PCR reactions were performed in duplicate. Agarose gel electrophoresis and melting curve analysis were used to verify the specificity of PCR products. Relative gene expression was calculated by normalizing the expression of each gene with GAPDH expression using the double delta $\mathrm{Ct}$ analysis.

\section{Migration Test}

To evaluate the effect of curcumin on hGF migration, after wounding, the cells were treated with vehicle (DMSO) or $1 \mu \mathrm{M}$ curcumin for 48 hours. Images of the wounded area

Table 1 Primer sequences used for qPCR

\begin{tabular}{|l|l|l|}
\hline Gene & Primer sequence & $\begin{array}{l}\text { Product } \\
\text { size }\end{array}$ \\
\hline COL1 & $\begin{array}{l}\text { Forward: } \\
\text { 5'-CCTCAAGGGCTCCAACGAG-3' } \\
\text { Reverse: } \\
\text { 5'-TCAATCACTGTCTTGCCCCA-3' }\end{array}$ & 117 \\
\hline KGF-1 & $\begin{array}{l}\text { Forward: } \\
\text { 5'-CCCTGAGCGACACACAAGAAGT-3' } \\
\text { Reverse: } \\
\text { 5'-CCACAATTCCAACTGCCACTG-3' }\end{array}$ & 182 \\
\hline EGFR & $\begin{array}{l}\text { Forward: } \\
\text { 5'-CCACCAAATTAGCCTGGACA-3' } \\
\text { Reverse: } \\
\text { 5'-CGCGACCCTTAGGTATTCTG-3' }\end{array}$ & 118 \\
\hline GAPDH & $\begin{array}{l}\text { Forward: } \\
\text { 5'-TGAACGGGAAGCTCACTGG- 3' } \\
\text { Reverse: } \\
\text { 5'-TCCACCACCCTGTTGCTGTA- 3' }\end{array}$ & \\
\hline
\end{tabular}

Abbreviations: COL1, collagen type I; EGFR, epidermal growth factor receptor; KCF 1, keratinocyte growth factor-1; qPCR, quantitative polymerase chain reaction GAPDH, glyceraldehyde-3-phosphate dehydrogenase. were captured at 0,24 , and 48 hours after curcumin treatment using photomicrograph $(\times 100)$ at the same culture plate position by marking the bottom of the plates. Three independent experiments were performed with GFs from three different patients $(n=9)$. The wound area in each photomicrograph was measured three times using ImageAnalysis J Software and the average wound area was calculated as percentage of the wound area at 0 hour.

\section{Statistical Analysis}

The data were reported as mean \pm standard deviation (SD) from three independent experiments with GFs from three different patients in each experimental condition $(n=9)$. The normality of the data was determined by the Shapiro-Wilk test. Data with normal distribution was analyzed by independent $t$-test or one-way analysis of variance (ANOVA) followed by Tukey's Honestly Significant Difference (HSD) test. Differences at $p<0.05$ were considered statistically significant.

\section{Results}

Curcumin Induced KGF-1 and EGFR Expressions in hGFs In unwounded GFs, curcumin at 0.1 to $20 \mu \mathrm{M}$ did not significantly affect COL1 mRNA expression ( contrast, curcumin at $1 \mu \mathrm{M}$ significantly increased KGF-1 and EGFR mRNA expressions $(p<0.05)$ while $0.1,1$, and $10 \mu \mathrm{M}$ had no significant effect ( $\mathbf{- F i g . 1 B}$ and $\mathbf{1 C}$ ). Therefore, $1 \mu \mathrm{M}$ curcumin was used in subsequent experiments.

\section{Curcumin Upregulated Wound Healing-Related Gene Expression in the In Vitro Wound Healing Model}

To investigate the effect of curcumin on expression of wound healing-related genes in the in vitro wound healing model, the hGF cell monolayer was wounded prior to $1 \mu \mathrm{M}$ curcumin treatment. After 24 hours, the mRNA expression of COL1, KGF-1, and EGFR was determined by quantitative PCR (qPCR). Curcumin significantly upregulated COL1, KGF-1, and EGFR mRNA expressions $(p<0.05)$ compared with the vehicle-treated control in the in vitro wound healing model ( - Fig. 2).

\section{Inhibiting ERK Signaling Attenuated Curcumin- Induced Gene Expression in the In Vitro Wound Healing Model}

To investigate the role of ERK in the curcumin-induced wound healing-related gene expression, wounded hGF monolayer were pretreated with PD98059, a MEK/ERK signaling pathway inhibitor, for 1 hour prior to curcumin treatment. PD98059 significantly decreased the curcumin-induced COL1 and EGFR mRNA expressions $(p<0.05)$ as shown in - Fig. $\mathbf{3 A}$ and $\mathbf{3 C}$. However, the curcumin-induced KGF-1 mRNA expression was not significantly affected by PD98059 pretreatment ( - Fig. 3B).

\section{Curcumin Did Not Significantly Affect Gingival Fibroblast Migration}

The scratch assay demonstrated that curcumin slightly accelerated the migration of hGFs into the wound area 
A

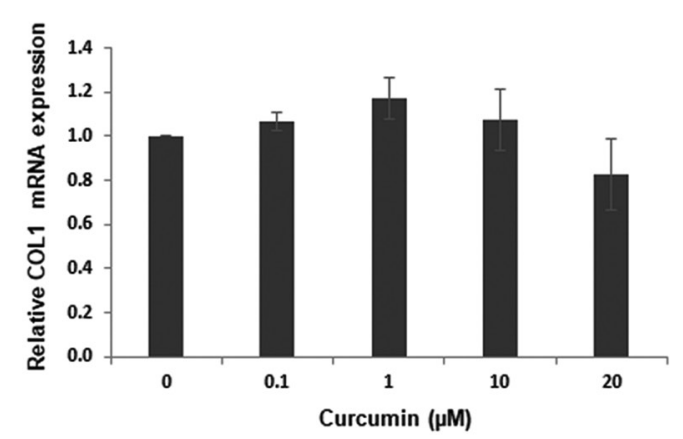

B

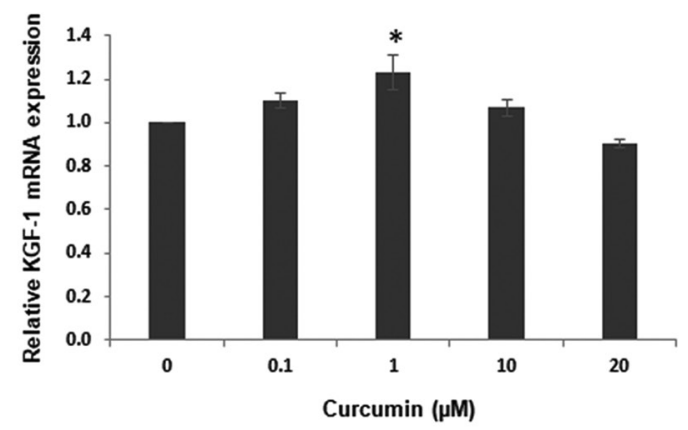

C

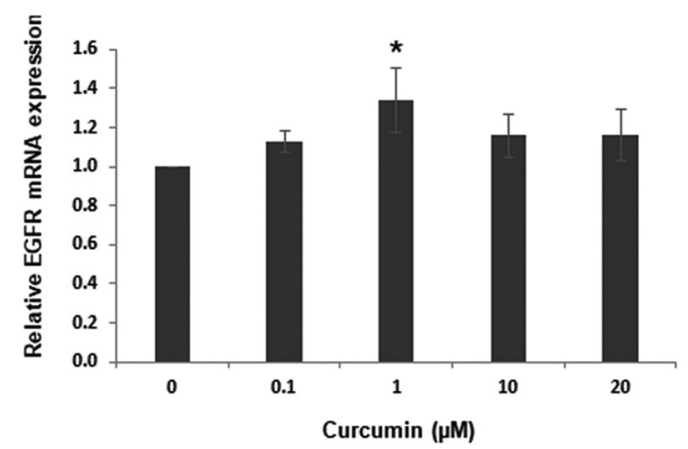

Fig. 1 The mRNA expression of COL1 (A), KGF-1 (B), and EGFR (C) in hGFs in response to curcumin. Cells were treated with varying concentrations of curcumin or DMSO for 24 hours. The level of gene expression was determined by $\mathrm{qPCR}$. The data are the mean \pm SD from three independent experiments in which each group has samples from three different patients $(n=9)$. Statistical analysis was performed by one-way ANOVA followed by Tukey's HSD test. *indicates compared with the control group $(p<0.05)$. ANOVA, analysis of variance; COL1, collagen type I; EGFR, epidermal growth factor receptor; hGFs, human gingival fibroblasts; HSD, honestly significant difference; KGF 1, keratinocyte growth factor-1; qPCR, quantitative polymerase chain reaction; SD, standard deviation.

compared with the vehicle-treated group at 24 hours ( - Fig. $4 \mathrm{~B}$ and $\mathbf{4 E}$ ) and 48 hours ( - Fig. $4 \mathrm{C}$ and $\mathbf{4 F}$ ). However, the remaining wound area in each group was not significantly different at both time points ( - Fig. 4G).

\section{Discussion}

In the present study, we investigated the effect of curcumin on the expression of COL1, KGF-1, and EGFR in hGFs. A previous study from our laboratory demonstrated that 0.1 to
A

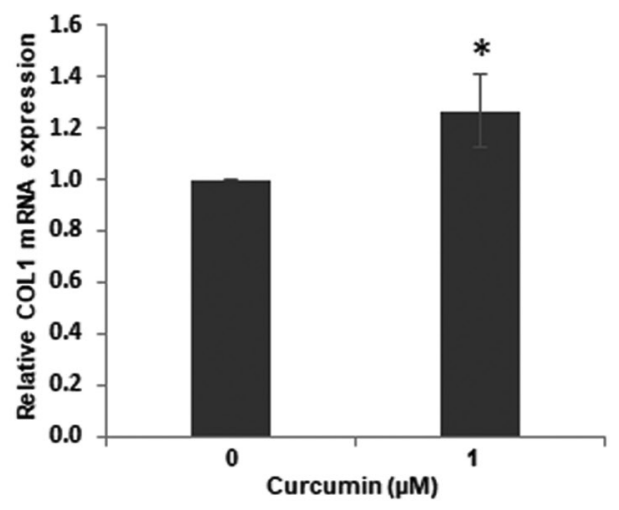

B

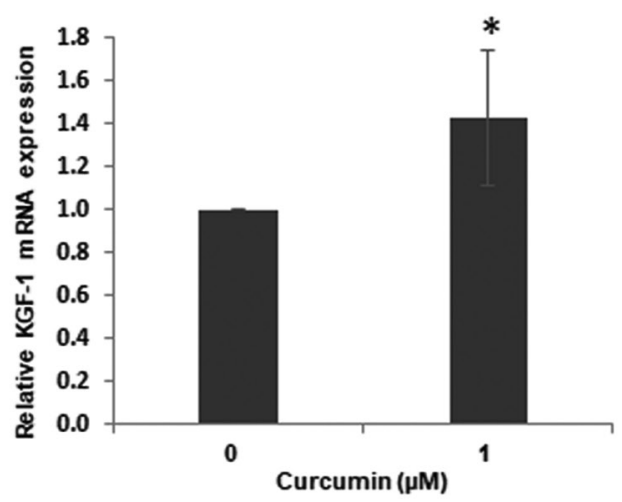

C

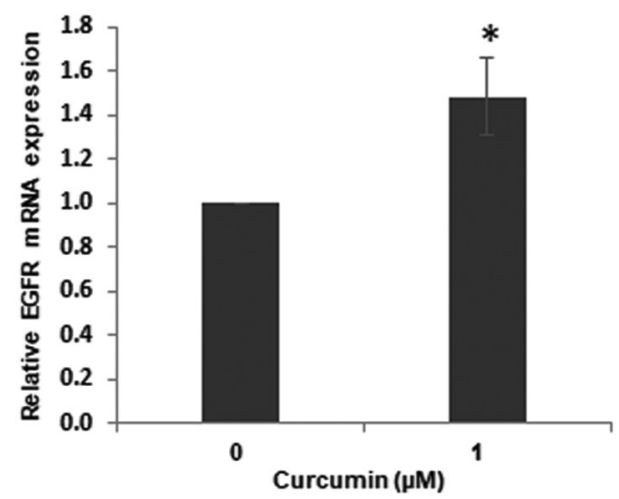

Fig. 2 The expression of COL1 (A), KGF-1 (B), and EGFR (C) in hGFs in response to curcumin in the in vitro wound healing model. The hGF monolayer was scratched using a sterilized pipette tip and then treated with $1 \mu \mathrm{M}$ of curcumin or DMSO for 24 hours. The level of gene expression was determined by qPCR. The data are the mean \pm SD from three independent experiments in which each group has samples from three different patients $(n=9)$. Statistical analysis was performed by independent $t$-test. ${ }^{*}$ indicates compared with the control group $(p<0.05)$. COL1, collagen type I; EGFR, epidermal growth factor receptor; hGFs, human gingival fibroblasts; KGF 1, keratinocyte growth factor-1; qPCR, quantitative polymerase chain reaction; SD, standard deviation. 
A

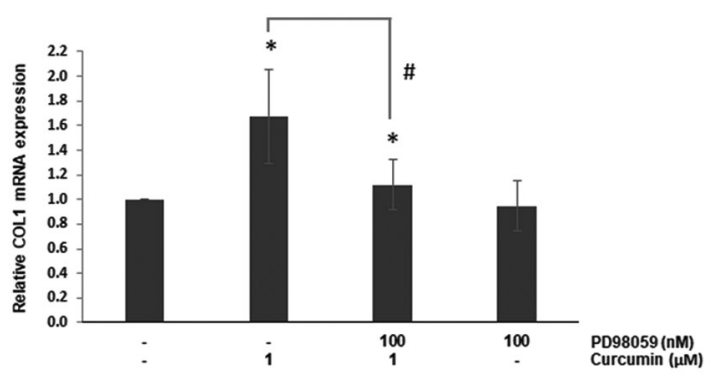

B

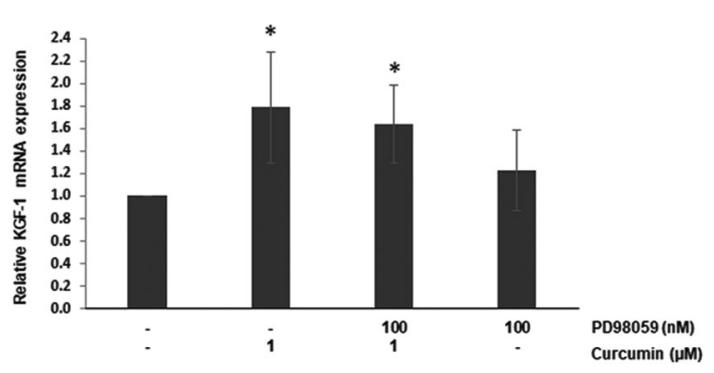

C

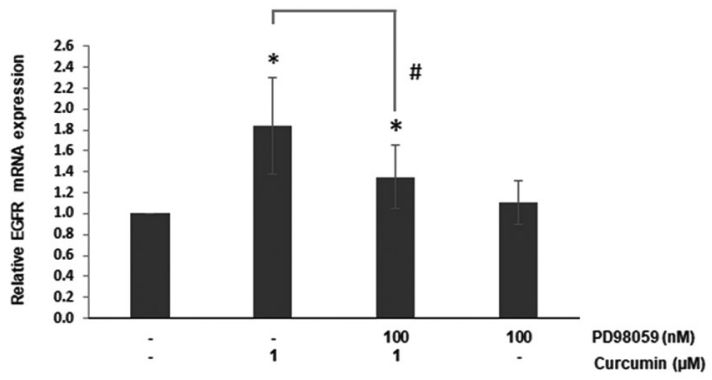

Fig. 3 Effect of inhibiting ERK signaling on the curcumin-induced COL1 (A), KGF-1 (B), and EGFR (C) expressions in hGFs in the in vitro wound healing model. The hGF monolayer was scratched and treated with $100 \mathrm{nM}$ PD98059 for 1 hour prior to $1 \mu \mathrm{M}$ curcumin for 24 hours. The level of gene expression was determined by GPCR. The data are the mean \pm SD from three independent experiments in which each group has samples from three different patients $(n=9)$. Statistical analysis was performed by one-way ANOVA followed by Tukey's HSD test. ${ }^{*}$ indicates compared with the control group $(p<0.05)$, \#indicates compared with curcumin-treated group $(p<0.05)$. ANOVA, analysis of variance; COL1, collagen type I; EGFR, epidermal growth factor receptor; hGFs, human gingival fibroblasts; HSD, honestly significant difference; KGF 1, keratinocyte growth factor-1; qPCR, quantitative polymerase chain reaction; SD, standard deviation.

$20 \mu \mathrm{M}$ curcumin had no significant effect on cell viability or cell proliferation, whereas higher concentrations of curcumin induced a significant dose-dependent cytotoxicity. Thus, 0.1 to $20 \mu \mathrm{M}$ curcumin was used in this study. A similar study reported that cell viability of primary hGFs was not affected by curcumin concentration up to $10 \mu \mathrm{M} .^{18}$

In unwounded GFs, only $1 \mu \mathrm{M}$ curcumin significantly increased KGF-1 and EGFR mRNA expressions, while 0.1, 10, and $20 \mu \mathrm{M}$ curcumin did not upregulate these genes. Even though COL1 mRNA expression was not significantly affected by 0.1 to $20 \mu \mathrm{M}$ curcumin treatment, $1 \mu \mathrm{M}$ curcumin exhibited a tendency to be the optimal concentration to induce COL1 mRNA expression in hGFs. These findings indicated that the effect of curcumin on gene expression in hGFs is not dose-dependent but requires the optimal concentration of curcumin. Our results suggest that curcumin regulated expression of wound healing-related genes such as KGF-1 and EGFR in unwounded hGFs.

Curcumin has been reported to promote oral wound healing in vivo; however, the mechanisms remain unclear. ${ }^{14,15}$ Though not an ideal wound healing model, the scratch assay has been widely used to study the functions of various molecules on cultured keratinocytes and fibroblasts. ${ }^{17,19}$ In the present study, the scratch assay was used to determine the effect of curcumin on hGF gene expression following injury. Interestingly, curcumin significantly upregulated COL1, KGF1 , and EFGR in this in vitro wound healing model. COL1 is the most abundant protein found in gingival tissues. Previous studies reported that collagen and collagen-derived fragments regulate many cellular functions, including cell shape and differentiation, migration, and synthesis of multiple proteins. ${ }^{20,21}$ An in vitro study showed that COL1 promoted human keratinocyte migration. ${ }^{22}$ Furthermore, collagen induced endothelial cell monolayers to reorganize into a network of branching and anastomosing capillary-like tubes. ${ }^{20}$ Collagen plays an important role in fibroblast recruitment to the wounded area, and regulation of collagen and matrix metalloproteinase (MMP) synthesis. ${ }^{21}$ Our finding that curcumin increased COL1 expression in hGFs is consistent with previous in vivo studies where curcumin stimulated collagen synthesis in irradiated wounds and corticosteroid-impaired abrasion wounds. ${ }^{23,24}$ These results suggest that curcumin might promote oral wound healing, partly by COL1 upregulation. Our findings that curcumin only stimulates COL1 expression in wounded hGFs but not in unwounded cells also suggest that curcumin preferentially upregulates COL1 when granulation tissue formation is required during wound healing. It remains unclear how curcumin regulates COL1 in these different settings but could be a result of fibroblast activation and different gene expression upon wounding. ${ }^{19,25}$ Mechanical injuries induce an increase of intracellular calcium leading to activation of multiple cellular process including gene expression. ${ }^{25}$ Curcumin has been reported to reduce the level of intracellular calcium release induced by chemical stimuli. ${ }^{26}$ Further investigations are required to investigate whether curcumin could modulate the release of intracellular calcium in response to mechanical injury.

KGF-1 binds to the keratinocyte growth factor receptor (KGFR), mainly expressed on keratinocytes and epithelial cells, with high affinity. KGF-1 controls several cellular processes, such as cell proliferation, differentiation, migration, and survival. KGF-1 stimulated keratinocyte proliferation and migration during the reepithelialization stage. ${ }^{27}$ In the present study, we report for the first time that curcumin significantly upregulates KGF-1 mRNA expression in hGFs, suggesting that curcumin could enhance reepithelialization during oral wound healing via increased KGF-1 expression.

EGF has an established role as an important factor in oral wound healing. ${ }^{28}$ EGF exerts its effect via binding to EGFR, a membrane-bound receptor tyrosine kinase that is localized on the cell surface of several cell types including keratinocytes and fibroblasts. ${ }^{29,30}$ EGF functions in a paracrine and autocrine manner to promote wound healing by increasing 
A

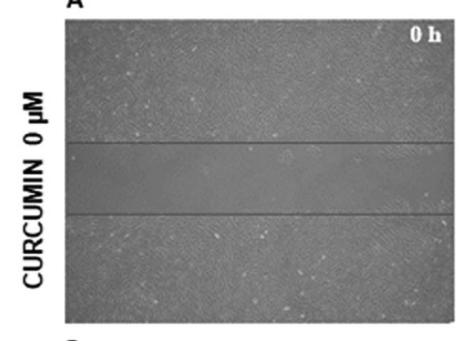

D

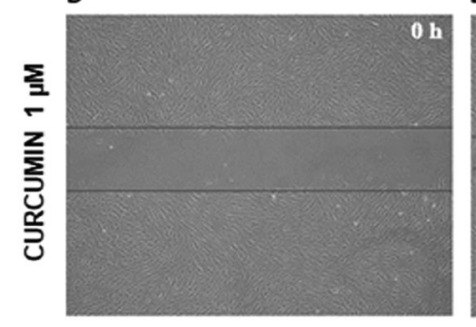

B

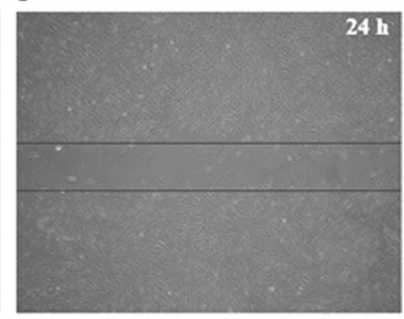

E

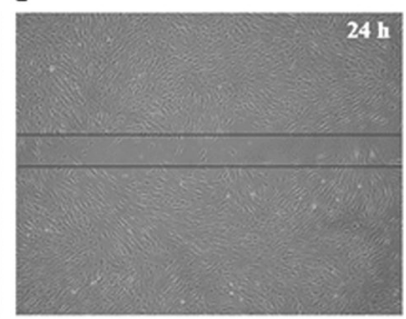

C

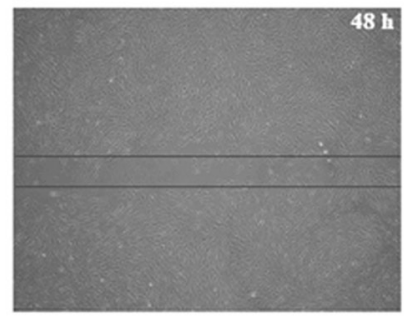

F

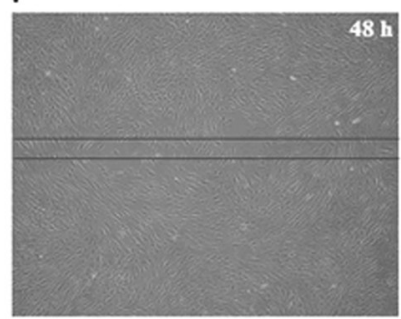

G

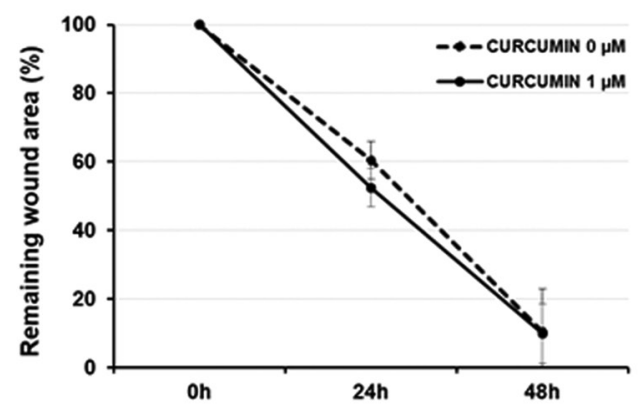

Fig. 4 Effect of curcumin on gingival fibroblast migration. The hGF monolayer was scratched and then treated with DMSO (A-C) or $1 \mu \mathrm{M}$ curcumin (D-F) for 24 to 48 hours. The images are representative of three separate experiments. (G) The remaining wound area at 0,24 , and 48 hours was analyzed by Image-Analysis J Software. The data are the mean \pm SD from three independent experiments in which each group has samples from three different patients $(n=9)$. Statistical analysis was performed by independent $t$-test. hGFs, human gingival fibroblasts; SD, standard deviation.

keratinocyte proliferation and migration. ${ }^{6,31}$ In addition to promoting reepithelialization, EGFR signaling stimulates fibroblast proliferation, differentiation, migration, and survival. ${ }^{30,32-34}$ In the present study, we demonstrated that curcumin increased EGFR expression in the in vitro wound healing model of hGFs suggesting that curcumin could promote wound healing by increasing the signal from the EGFR-binding growth factors. However, further studies are necessary to investigate whether the downstream signaling of EGFR activation is elevated by curcumin treatment.

EGFR is essential for stimulating migration of fibroblasts toward multiple extracellular matrix components via an integrin-dependent pathway. ${ }^{30}$ EGFR expression is also required for fibroblast migration stimulated by plateletderived growth factor (PDGF) which is a critical cytokine in the beginning stage of wound healing. ${ }^{35}$ In animal studies, curcumin-treated wounds showed early migration of fibroblasts and myofibroblasts into the wound bed. ${ }^{36}$ However, our results demonstrated that curcumin did not have a significant effect on hGF migration in vitro, which is consistent with a previous study where curcumin did not affect NIH3T3 fibroblast migration. ${ }^{37}$ Our findings that curcumin increased EGFR expression in wounded hGFs raised a question whether EGFR upregulation by curcumin could promote PDGF-induced and extracellular matrix-dependent fibroblast migration in vivo. Further investigation is required to explore this possibility, which might explain the contradictory results between the effect of curcumin on fibroblast migration in vitro and the in vivo wound healing process that involved several growth factors and the extracellular matrix.

Curcumin regulates gene expression by several mechanisms including modulating enzyme activity, such as protein kinases. ${ }^{16}$ The mitogen-activated protein kinase (MAPK) pathway has been shown to play an important role in the transduction of extracellular signals to cellular responses. ${ }^{38}$ MAPK plays a key role in many cellular functions including cell proliferation, differentiation, and apoptosis. The MAPK family consists extracellular signal-regulated kinase (ERK), C-Jun N-terminal kinase/stress activated protein kinase (JNK/ SAPK), and p38 MAPK. ${ }^{38}$ The ERK signaling pathway has an established role in cutaneous and corneal epithelial wound healing. ${ }^{25}$ Our data revealed that PD98059, an ERK signaling inhibitor, significantly decreased curcumin-induced COL1 and EGFR mRNA expressions, but did not have a significant effect on KGF-1 mRNA expression. To our best knowledge, we 
demonstrated for the first time that curcumin regulation of COL1 and EGFR expressions in hGFs requires ERK activation.

\section{Conclusion}

In the present study, we demonstrated that curcumin significantly upregulated the mRNA expression of COL1, KGF-1, and EGFR in hGFs in the in vitro wound healing model. ERK signaling is essential for curcumin-induced COL1 and EGFR mRNA expressions. Our results have expanded the insight into the complex mechanism of curcumin in regulation of wound healing-related gene expression. Further in vitro and clinical studies are necessary to understand how curcumin promotes oral wound healing.

\section{Ethical Approval}

The study protocol was approved by the Ethics Committee of the Faculty of Dentistry, Chulalongkorn University.

\section{Funding}

This study was supported by the Faculty Research Grant (DRF62007), Faculty of Dentistry, Chulalongkorn University, Thailand.

\section{Conflict of Interest}

None declared.

\section{Acknowledgment}

The authors thank Dr. Kevin Tompkins for his proofreading of the manuscript.

\section{References}

1 Brand HS, Ligtenberg AJ, Veerman EC. Saliva and wound healing. Monogr Oral Sci 2014;24:52-60

2 Yang C, Hillas PJ, Báez JA, et al. The application of recombinant human collagen in tissue engineering. BioDrugs 2004;18(2):103-119

3 Diegelmann RF. Analysis of collagen synthesis. Methods Mol Med 2003;78:349-358

4 Finch PW, Rubin JS. Keratinocyte growth factor/fibroblast growth factor 7, a homeostatic factor with therapeutic potential for epithelial protection and repair. Adv Cancer Res 2004; 91:69-136

5 Oxford GE, Nguyen KHT, Alford CE, Tanaka Y, Humphreys-Beher MG. Elevated salivary EGF levels stimulated by periodontal surgery. J Periodontol 1998;69(4):479-484

6 Schultz G, Rotatori DS, Clark W. EGF and TGF-alpha in wound healing and repair. J Cell Biochem 1991;45(4):346-352

7 Wells A. EGF receptor. Int J Biochem Cell Biol 1999;31(6): 637-643

8 Repertinger SK, Campagnaro E, Fuhrman J, El-Abaseri T, Yuspa SH, Hansen LA. EGFR enhances early healing after cutaneous incisional wounding. J Invest Dermatol 2004;123(5): 982-989

9 Sharma RA, Euden SA, Platton SL, et al. Phase I clinical trial of oral curcumin: biomarkers of systemic activity and compliance. Clin Cancer Res 2004;10(20):6847-6854

10 Meng B, Li J, Cao H. Antioxidant and antiinflammatory activities of curcumin on diabetes mellitus and its complications. Curr Pharm Des 2013;19(11):2101-2113

11 Tyagi P, Singh M, Kumari H, Kumari A, Mukhopadhyay K. Bactericidal activity of curcumin I is associated with damaging of bacterial membrane. PLoS One 2015;10(3):e0121313
12 Aggarwal S, Takada Y, Singh S, Myers JN, Aggarwal BB. Inhibition of growth and survival of human head and neck squamous cell carcinoma cells by curcumin via modulation of nuclear factor-kappaB signaling. Int J Cancer 2004;111(5):679-692

13 Akbik D, Ghadiri M, Chrzanowski W, Rohanizadeh R. Curcumin as a wound healing agent. Life Sci 2014;116(1):1-7

14 Lim YS, Kwon SK, Park JH, Cho CG, Park SW, Kim WK. Enhanced mucosal healing with curcumin in animal oral ulcer model. Laryngoscope 2016;126(2):E68-E73

15 Manifar S, Obwaller A, Gharehgozloo A. Boorboor Shirazi Kordi $\mathrm{H}$, Akhondzadeh S. Curcumin gel in the treatment of minor aphthous ulcer: A randomized, placebo-controlled trial. Faslnamah-i Giyahan-i Daruyi 2012;1(41):40-45

16 Shishodia S. Molecular mechanisms of curcumin action: gene expression. Biofactors 2013;39(1):37-55

17 Riahi R, Long M, Yang Y, et al. Single cell gene expression analysis in injury-induced collective cell migration. Integr Biol 2014;6(2):192-202

18 Chen JT, Wang CY, Chen MH. Curcumin inhibits TGF-1-induced connective tissue growth factor expression through the interruption of Smad2 signaling in human gingival fibroblasts. J Formos Med Assoc 2018;117(12):1115-1123

19 Häkkinen L, Uitto VJ, Larjava H. Cell biology of gingival wound healing. Periodontol 2000 2000;24:127-152

20 Montesano R, Orci L, Vassalli P. In vitro rapid organization of endothelial cells into capillary-like networks is promoted by collagen matrices. J Cell Biol 1983;97(5 Pt 1) :1648-1652

21 Leitinger B, Hohenester E. Mammalian collagen receptors. Matrix Biol 2007;26(3):146-155

22 Woodley DT, Bachmann PM, O'Keefe EJ. Laminin inhibits human keratinocyte migration. J Cell Physiol 1988;136(1):140-146

23 Bhagavathula N, Warner RL, DaSilva M, et al. A combination of curcumin and ginger extract improves abrasion wound healing in corticosteroid-impaired hairless rat skin. Wound Repair Regen 2009;17(3):360-366

24 Jagetia GC, Rajanikant GK. Role of curcumin, a naturally occurring phenolic compound of turmeric in accelerating the repair of excision wound, in mice whole-body exposed to various doses of gamma-radiation. J Surg Res 2004;120(1):127-138

25 Ghilardi SJ, O'Reilly BM, Sgro AE. Intracellular signaling dynamics and their role in coordinating tissue repair. Wiley Interdiscip Rev Syst Biol Med 2020;12(3):e1479

26 Chen YC, Kuo TC, Lin-Shiau SY, Lin JK. Induction of HSP70 gene expression by modulation of $\mathrm{Ca}(+2)$ ion and cellular $\mathrm{p} 53$ protein by curcumin in colorectal carcinoma cells. Mol Carcinog 1996;17(4):224-234

27 Sogabe Y, Abe M, Yokoyama Y, Ishikawa O. Basic fibroblast growth factor stimulates human keratinocyte motility by Rac activation. Wound Repair Regen 2006;14(4):457-462

28 Noguchi S, Ohba Y, Oka T. Effect of salivary epidermal growth factor on wound healing of tongue in mice. Am J Physiol 1991;260(4 Pt 1) :E620-E625

29 Seeger MA, Paller AS. The roles of growth factors in keratinocyte migration. Adv Wound Care (New Rochelle) 2015;4(4): 213-224

30 Li J, Lin ML, Wiepz GJ, Guadarrama AG, Bertics PJ. Integrinmediated migration of murine B82L fibroblasts is dependent on the expression of an intact epidermal growth factor receptor. J Biol Chem 1999;274(16):11209-11219

31 Hudson LG, McCawley LJ. Contributions of the epidermal growth factor receptor to keratinocyte motility. Microsc Res Tech 1998;43(5):444-455

32 Wieduwilt MJ, Moasser MM. The epidermal growth factor receptor family: biology driving targeted therapeutics. Cell Mol Life Sci 2008;65(10):1566-1584

33 Hollenberg MD, Cuatrecasas P. Epidermal growth factor: receptors in human fibroblasts and modulation of action by cholera toxin. Proc Natl Acad Sci U S A 1973;70(10):2964-2968 
34 Kim D, Kim SY, Mun SK, Rhee S, Kim BJ. Epidermal growth factor improves the migration and contractility of aged fibroblasts cultured on 3D collagen matrices. Int J Mol Med 2015;35(4):1017-1025

35 Li J, Kim YN, Bertics PJ. Platelet-derived growth factor-stimulated migration of murine fibroblasts is associated with epidermal growth factor receptor expression and tyrosine phosphorylation. J Biol Chem 2000;275(4):2951-2958
36 Sidhu GS, Singh AK, Thaloor D, et al. Enhancement of wound healing by curcumin in animals. Wound Repair Regen 1998;6(2):167-177

37 Topman G, Lin FH, Gefen A. The natural medications for wound healing - curcumin, aloe-vera and ginger - do not induce a significant effect on the migration kinematics of cultured fibroblasts. J Biomech 2013;46(1):170-174

38 Zhang W, Liu HT. MAPK signal pathways in the regulation of cell proliferation in mammalian cells. Cell Res 2002;12(1):9-18 